ISSN 2447-9071

doi $10.36414 /$ rbmc.v5i12.5
Contato para correspondência: Roberpaulo Anacleto Neves

E-mail:

roberpaulo_@hotmail.com

Conflito de interesse: Não

Financiamento: Recursos próprios

Recebido: 15/06/2019

Aprovado: 19/07/2019

\section{Violência sexual infanto-juvenil no estado de Goiás: análise epidemiológica}

\author{
Sexual violence children and youth in the state of Goiás: \\ epidemiological analysis
}

\author{
Helen Cristina Fernandes ${ }^{1}$, Danilo Martins Rosa Costa ${ }^{2}$, Roberpaulo Anacleto Neves ${ }^{3}$ \\ 'Médica, Pontifícia Universidade Católica de Goiás, ${ }^{2}$ Médico, Pontifícia Universidade Católica \\ de Goiás, ${ }^{3}$ Biomédico, Mestre em Ciências Ambientais e Saúde pela PUC Goiás, Doutorando \\ em Biotecnologia e Biodiversidade pela UnB, Professor na Pontifícia Universidade Católica de \\ Goiás, Professor da Faculdade da Polícia Militar.
}

\begin{abstract}
Resumo
A violência sexual contra crianças e adolescentes é um problema de saúde pública, caracterizado por uma das formas mais graves de violação aos direitos humanos. O objeto deste estudo foi analisar o perfil das vítimas de violência sexual, contra crianças e adolescentes, no intuito de modificar a realidade vigente através de medidas e ações de saúde públicas voltadas para esse público. Trata-se de um estudo descritivo retrospectivo observacional, com abordagem quantitativa com dados coletados no sistema de vigilância de violência e acidentes (VIVA), a partir das notificações relacionadas aos tipos de violência sexuais referentes aos anos de 2013 e 2014, no Estado de Goiás, na faixa etária de 0 a 19 anos, sendo estes dados tabulados pelo Microsoft Excel ${ }^{\circledR}$ e pelo software estatístico, SPSS v. 23. Foram observadas 4.635 notificações de casos de violência sexual no Estado de Goiás nos anos de 2013 e 2014, com maior prevalência no sexo feminino, sendo a raça parda a mais acometida e a residência o local de ocorrência mais comum. De modo geral, os resultados do presente estudo evidenciam que a violência sexual praticada contra crianças e adolescentes no estado de Goiás nos anos de 2013 e 2014, predomina, em números absolutos, o estupro, seguido da violência psicomoral, sendo o local mais comum no ambiente doméstico, a cor/raça sendo a parda e o sexo feminino como maioria das vítimas. A violência sexual contra crianças e adolescentes é um tema que tem atraído a atenção de governantes, estudiosos, bem como da sociedade geral.
\end{abstract}

Palavras-Chave: Abuso sexual, crianças, adolescentes, perfil epidemiológico.

\begin{abstract}
Sexual violence against children and adolescents is a public health problem characterized by one of the most serious forms of human rights violations. The objective of this study was to analyze the profile of victims of sexual violence against children and adolescents, in order to modify the current reality through public health measures and actions aimed at this public. This is a descriptive retrospective observational study, with a quantitative approach with data collected in the violence and accident surveillance system (VIVA), based on reports related to the types of sexual violence related to the years 2013 and 2014, in the state of Goiás, in the age group from 0 to 19 years, these data being tabulated by Microsoft Excel ${ }^{\oplus}$ and by statistical software, SPSS v. 23. There were 4,635 reports of cases of sexual violence in the State of Goiás in the years of 2013 and 2014, with a higher prevalence in females, being the brown race the most affected and the residence the most common place of occurrence. In general, the results of the present study show that sexual violence practiced against children and adolescents in the state of Goiás in the years of 2013 and 2014, in absolute numbers, rape, followed by psychomoral violence, is the most common place in the domestic environment, color/race being brown and female as the majority ofvictims. Sexual violence against children and adolescents is a topic that has attracted the attention of rulers, scholars, and society as a whole.
\end{abstract}

Palavras-Chave: Sexual abuse, children, adolescents, epidemiological profile. 


\section{Introdução}

A violência é uma relação assimétrica de poder, em que o sujeito agressor se encontra em posição superior cometendo um dano; seja ele físico, sexual, psicológico, contrário à vontade da vítima, por um mecanismo que anule sua vontade pessoal'.

No Brasil, considera-se violência contra criança, quando esta se encontra na faixa etária de zero a 12 anos de idade incompletos, e adolescente entre 12 e 19 anos incompletos, conforme o que dispõe o Estatuto da Criança e Adolescente (ECA - Lei 8.069 de 13 de julho de 1990)². Divisão categórica estritamente objetiva, dando conceito legal, sendo que outras ciências podem adotar parâmetros etários diversos.

Violência sexual contra crianças e adolescentes é o envolvimento destes em atividades sexuais com um adulto, ou com qualquer pessoa um pouco mais velha ou maior, nas quais haja uma diferença de idade, de tamanho ou de poder, em que a criança é usada como objeto sexual para gratificação das necessidades ou dos desejos do agressor, sendo ela incapaz de dar um consentimento consciente por causa do desequilíbrio no poder ou de qualquer incapacidade mental ou física ${ }^{3}$.

$A$ violência sexual não ocorre apenas ao estupro e à violência física, mas também por uma série de atividades que não envolvem contato físico, como telefonemas obscenos, exibicionismo, voyeurismo, aliciamento pela internet, estímulo à nudez, dentre outros. A violência sexual é um evento mórbido que vitimiza crianças e adolescentes e que se transformou em um problema de saúde pública ${ }^{4}$.

Apesar de sua crescente incidência e da importância do diagnóstico para que se tenha a implementação de programas e ações preventivas baseadas nos fatores de risco, a violência sexual continua sendo o tipo de violência contra crianças e adolescentes de menor notificação. Isso se traduz por diversos motivos, como tabus culturais, medo do agressor, vergonha, falsa sensação de culpa, contribuindo para o desconhecimento sobre seus mecanismos e real incidência ${ }^{5}$, porém em 2009, a partir da inserção de dados no Sistema de informação de Agravos de Notificação (SINAN) a notificação de violência doméstica, sexual e outras violências apresentou progressiva expansão. No estado de Goiás no ano de 2013 o SINAN do Sistema Único de Saúde fez 631 registros de violência sexual contra menores de 18 anos.

No ano de 2014, 51 meninos e meninas foram internados em decorrência de agressão física e violência sexual. Os Números são elevados e refletem apenas uma face da grave questão já que grande parte dos casos de violência infantojuvenil não é divulgada, já que muitas vezes o autor da violência encontra-se no seio familiar ${ }^{5}$.

Conforme a análise dos dados do Mapa da Violência contra Crianças e Adolescentes do ano de 2011, se verificou que a maior parte das vítimas de violência é do sexo feminino, atingindo o índice $60,3 \%$ dos casos de violência em geral, enquanto que a violência contra crianças e adolescentes do sexo masculino representou $39,7 \%$ dos casos $^{6}$.

Em relação ao local da ocorrência da violência, a maior parte dos casos de notificação aconteceu no ambiente familiar, ou seja, foram registrados 21.041 casos nas residências, o que representa o percentual de $63,7 \%$, quantidade extremamente considerável, representando que os maiores agressores de crianças e adolescentes são os membros da família e pessoas próximas ao convívio familiar. Em segundo lugar com maior número de ocorrências se deu na via pública, atingindo o percentual de $18,1 \%$ dos casos $^{7}$. Pelo fato de os familiares exercerem alguma forma de poder ou dependência, é difícil a suspeita e complicada confirmação.

Conforme a legislação constitucional e infraconstitucional, os familiares têm o dever de proteger os direitos das crianças e adolescentes. Qualquer tipo de violência no ambiente familiar causa inúmeros prejuízos às vítimas, às vezes sequelas irreparáveis, deixando marcas traumáticas em seu desenvolvimento psicossocial ${ }^{8}$.

Normalmente esse tipo de violência se dá de forma insidiosa, em um ambiente relacional favorável, em que a criança não tenha consciência do ato abusivo, em um primeiro momento. $\mathrm{O}$ abusador coloca a criança ou adolescente como sedutor e que quer participar do abuso, fazendo a vítima se sentir culpada, inibindo ainda mais o desejo de denunciar?.

Trata-se de um problema de saúde pública com inúmeras implicações no desenvolvimento cognitivo-comportamental das crianças e adolescentes. Neste contexto o objeto deste estudo foi analisar o perfil das vítimas de violência sexual, contra crianças e adolescentes, no intuito de modificar a realidade vigente através de medidas e ações de saúde públicas voltadas para esse público.

\section{Métodos}

Trata-se de um estudo descritivo observacional retrospectivo. Neste estudo não ocorre nenhuma manipulação do fenômeno estudado. É uma investigação retrospectiva observacional em que o desfecho já ocorreu no passado.

Como critérios de inclusão, foram pesquisados todos os casos notificados de violência sexual em crianças e adolescentes inscritos no banco de dados do Sistema de Vigilância de Violências e Acidentes - VIVA, do período de 2013 e 2014, na faixa etária de 0 a 19 anos e inseridos no Estado de Goiás. Foram analisados os casos de estupro, atentado violento ao pudor, violência psicomoral, assédio sexual, pornografia infantil e exploração sexual. 
Como critérios de exclusão, ficaram de fora da pesquisa qualquer dado que tenha sido gerado em data conflitante com o período determinado para o estudo, idade das vítimas acima de 19 anos e outros tipos de violência que não a sexual.

Os dados coletados foram primeiramente tabulados em uma planilha com a utilização do software Excel (Office 2013) e posteriormente analisados com o auxílio do pacote estatístico SPSS, versão 23.0 (Statistical Package for Social Science). O nível de significância adotado foi de $5 \%(p<0,05)$.

Foram realizadas estatísticas descritivas em todas as variáveis, frequência absoluta e relativa, incluindo o teste do Qui-quadrado de Pearson ${ }^{10}$ ou Qui-quadrado Post hoc nas situações em que a contingência foi superior à $2 \times 2$. Essas análises possibilitaram a comparação das ocorrências em cada ano de acordo com o sexo, raça/cor e tipo de violência.

\section{Resultados}

Foram comunicadas 4.635 notificações realizadas no estado de Goiás, entre os anos de 2013 e 2014, para a faixa etária entre 0 e 19 anos, para os tipos de violência sexual: estupro, atentado violento ao pudor, violência psíquico moral, pornografia infantil, exploração sexual e assédio sexual. Quanto ao sexo, em 2013 foram notificados 2.337 casos de violência sexual contra crianças e adolescentes, dos quais $55,7 \%$ foram do sexo feminino e em 2014 teve um total de 2.296 casos notificados, sendo $44,6 \%$ do sexo feminino.

Em relação ao tipo de violência sexual, no período de 2013 e 2014, foram notificados 264 casos de assédio sexual, 797 casos de estupro, 756 casos de violência psicomoral, 38 casos de pornografia infantil, 56 casos de exploração sexual e 77 casos de atentado violento ao pudor.

Quanto aos tipos de violência sexual e as locais de ocorrência (Tabela 1), verificou-se que a residência foi o local mais comum de ocorrência, tanto em 2013 quanto em 2014 para todos os tipos de violência sexual. A residência, como local mais comum, no período, representou $69,4 \%$ nos casos de assédio sexual, $68,0 \%$ nos casos de atentado violento ao pudor, $58,6 \%$ nos casos de estupro, $69,2 \%$ nos casos de exploração sexual, $76,5 \%$ nos casos de pornografia e $62,7 \%$ nos casos de violência psicomoral. $O$ segundo local mais frequente foi a via pública e o terceiro foi a escola. Os locais classificados como "outros" foram: comércio/serviços, bar ou similar, local de prática esportiva, indústria, habitação coletiva, outros, em branco e ignorados. Estes foram agrupados, pois isoladamente, em números absolutos.
Tabela 1. Tipos de violência sexual em relação ao local de ocorrência, entre os períodos de 2013 e 2014.

\begin{tabular}{|c|c|c|c|c|}
\hline \multirow{2}{*}{$\begin{array}{l}\text { Local de } \\
\text { ocorrência }\end{array}$} & \multicolumn{2}{|c|}{ Ano n (\%) } & \multirow[b]{2}{*}{ Total } & \multirow[b]{2}{*}{$p^{*}$} \\
\hline & 2013 & 2014 & & \\
\hline \multicolumn{5}{|c|}{ Assédio sexual } \\
\hline Escola & $3(2,8)$ & $4(5,4)$ & $7(3,9)$ & 0,38 \\
\hline Residência & $72(67,9)$ & $53(71,6)$ & $125(69,4)$ & 0,39 \\
\hline Via pública & $10(9,4)$ & $6(8,1)$ & $16(8,9)$ & 0,60 \\
\hline Outros & $21(19,8)$ & $11(14,9)$ & $32(17,8)$ & 0,76 \\
\hline Total & 106 & 74 & 180 & \\
\hline \multicolumn{5}{|c|}{ Atentado violento ao pudor } \\
\hline Escola & $2(3,6)$ & $1(2,3)$ & $3(3,0)$ & 0,71 \\
\hline Residência & $40(71,4)$ & $28(63,6)$ & $68(68,0)$ & 0,84 \\
\hline Via pública & $1(1,8)$ & $4(9,1)$ & $5(5,0)$ & 0,41 \\
\hline Outros & $13(23,2)$ & $11(25,0)$ & $24(24,0)$ & 0,10 \\
\hline Total & 56 & 44 & 100 & \\
\hline \multicolumn{5}{|l|}{ Estupro } \\
\hline Escola & $2(0,5)$ & $9(2,9)$ & $11(1,6)$ & $0,02 *$ \\
\hline Residência & $215(58,1)$ & $184(59,2)$ & $399(58,6)$ & 0,35 \\
\hline Via pública & $62(16,8)$ & $51(16,4)$ & $113(16,6)$ & 0,78 \\
\hline Outros & $91(24,6)$ & $67(21,5)$ & $158(23,2)$ & 0,90 \\
\hline Total & 370 & 311 & 681 & \\
\hline \multicolumn{5}{|c|}{ Exploração sexual } \\
\hline Escola & $1(4,3)$ & $0(0,0)$ & $1(2,6)$ & 0,40 \\
\hline Residência & $17(73,9)$ & $10(62,5)$ & $27(69,2)$ & 0,56 \\
\hline Via pública & $1(4,3)$ & $2(12,5)$ & $3(7,7)$ & 0,45 \\
\hline Outros & $4(17,4)$ & $4(25,0)$ & $8(20,5)$ & 0,35 \\
\hline Total & 23 & 16 & 39 & \\
\hline \multicolumn{5}{|c|}{ Pornografia infantil } \\
\hline Residência & $16(84,2)$ & $10(66,7)$ & $26(76,5)$ & 0,10 \\
\hline Via pública & $1(5,3)$ & $0(0,0)$ & $1(2,9)$ & 0,23 \\
\hline Outros & $2(10,5)$ & $5(33,3)$ & $7(20,6)$ & 0,37 \\
\hline Total & 19 & 15 & 34 & \\
\hline \multicolumn{5}{|c|}{ Violência psicomoral } \\
\hline Escola & $31(7,7)$ & $12(4,8)$ & $43(6,6)$ & 0,15 \\
\hline Residência & $259(64,1)$ & $151(60,4)$ & $410(62,7)$ & 0,42 \\
\hline Via pública & $54(13,4)$ & $44(17,6)$ & $98(15,0)$ & 0,34 \\
\hline
\end{tabular}




\begin{tabular}{lcccc} 
Outros & $60(14,9)$ & $43(17,2)$ & $103(15,7)$ & $\mathbf{0 , 1 4}$ \\
Total & 404 & 250 & 654 & \\
\hline
\end{tabular}

*Qui-quadrado Posthoc

Em relação ao tipo de violência e ao sexo (Tabela 2), verificou-se que o sexo feminino foi o mais comum, tanto em 2013 quanto em 2014, para todos os tipos de violência sexual. Crianças e adolescentes mulheres representaram $76,0 \%$ dos casos de assédio sexual, $80,5 \%$ dos casos de atentado violento ao pudor, $85,5 \%$ dos casos de estupro, $75,0 \%$ dos casos de exploração sexual, $82,1 \%$ dos casos de pornografia e $72,0 \%$ dos casos de violência psicomoral.

Tabela 2. Tipos de violência sexual em relação ao sexo, entre os períodos de 2013 e 2014.

\begin{tabular}{|c|c|c|c|c|}
\hline \multirow{2}{*}{ Local de ocorrência } & \multicolumn{2}{|c|}{ Ano n (\%) } & \multirow[b]{2}{*}{ Total } & \multirow[b]{2}{*}{$p^{*}$} \\
\hline & 2013 & 2014 & & \\
\hline \multicolumn{5}{|l|}{ Assédio sexual } \\
\hline Feminino & $79(77,5)$ & $48(73,8)$ & $127(76,0)$ & 0,59 \\
\hline Masculino & $23(22,5)$ & $17(26,2)$ & $40(24,0)$ & \\
\hline \multirow[t]{2}{*}{ Total } & 102 & 65 & 167 & \\
\hline & $21(19,8)$ & $11(14,9)$ & $32(17,8)$ & 0,76 \\
\hline \multicolumn{5}{|c|}{ Atentado violento ao pudor } \\
\hline Feminino & $34(79,1)$ & $28(82,4)$ & $62(80,5)$ & 0,72 \\
\hline Masculino & $9(20,9)$ & $6(17,6)$ & $15(19,5)$ & \\
\hline Total & 43 & 34 & 77 & \\
\hline \multicolumn{5}{|l|}{ Estupro } \\
\hline Feminino & $247(84,3)$ & $226(86,9)$ & $473(85,5)$ & 0,38 \\
\hline Masculino & $46(15,7)$ & $34(13,1)$ & $80(14,5)$ & \\
\hline \multirow[t]{2}{*}{ Total } & 293 & 260 & 553 & \\
\hline & $2(0,5)$ & $9(2,9)$ & $11(1,6)$ & $0,02^{*}$ \\
\hline \multicolumn{5}{|l|}{ Exploração sexual } \\
\hline Feminino & $16(80,0)$ & $8(66,7)$ & $24(75,0)$ & 0,40 \\
\hline Masculino & $4(20,0)$ & $4(33,3)$ & $8(25,0)$ & \\
\hline Total & 20 & 12 & 32 & \\
\hline \multicolumn{5}{|l|}{ Pornografia infantil } \\
\hline Feminino & $14(82,4)$ & $9(81,8)$ & $23(82,1)$ & 0,97 \\
\hline Masculino & $3(17,6)$ & $2(18,2)$ & $5(17,9)$ & \\
\hline Total & 17 & 11 & 28 & \\
\hline
\end{tabular}

\begin{tabular}{lcccc} 
Violência psicomoral & & & \\
Feminino & $286(70,8)$ & $185(74,0)$ & $471(72,0)$ & $\mathbf{0 , 3 7}$ \\
Masculino & $118(29,2)$ & $65(26,0)$ & $183(28,0)$ & \\
Total & 404 & 250 & 654 \\
\hline
\end{tabular}

${ }^{*}$ Qui-quadrado de Pearson

De acordo com a distribuição do total de violência sexual de acordo com a cor/raça no período de 2013 e 2014 (Tabela 3), verificou-se que a raça parda foi a mais prevalente, seguida da branca, depois amarelo/indígena e por último da preta.

Tabela 3. Distribuição do total de violência sexual de acordo com a cor/raça, comparando entre os períodos de 2013 e 2014.

\begin{tabular}{lcccc}
\hline \multirow{2}{*}{ Local de ocorrência } & 2013 & 2014 & Total & $p^{*}$ \\
Branco & $350(28,7)$ & $244(29,8)$ & $594(29,2)$ & 0,61 \\
Pardo & $525(43,1)$ & $376(45,9)$ & $901(44,2)$ & 0,21 \\
& $92(7,6)$ & $59(7,2)$ & $151(7,4)$ & 0,77 \\
Preto & & & & \\
Outros & $251(20,6)$ & $140(17,1)$ & $391(19,2)$ & 0,05 \\
Total & 1218 & 819 & 2037 & \\
\hline
\end{tabular}

*Qui-quadrado Posthoc

Quanto à comparação entre a cor/raça e o local de ocorrência da violência sexual no período de 2013 e 2014 (Tabela 4), verificou-se que, em 2013, nas raças amarelo/indígena, parda e preta o local de maior ocorrência da violência sexual foi a residência, exceto na raça branca, ficando em segundo lugar. Já em 2014, a residência como local de ocorrência mais prevalente foi comum a todas as raças.

Tabela 4. Comparação entre a cor/raça e o local de ocorrência nos períodos de 2013 e 2014.

\begin{tabular}{|c|c|c|c|c|c|}
\hline \multirow{2}{*}{ Local de ocorrência } & \multicolumn{4}{|c|}{ Cor / raça n (\%) } & \multirow[b]{2}{*}{ Total } \\
\hline & $\begin{array}{l}\text { Amarelo / } \\
\text { Indígena }\end{array}$ & Branco & Parda & Preta & \\
\hline \multicolumn{6}{|l|}{2013} \\
\hline Escola & $2(14,3)$ & $13(3,8)$ & $24(5,6)$ & $0(0,0)$ & $39(4,5)$ \\
\hline Residência & $9(64,3)$ & $67(19,5)$ & $238(56,0)$ & $62(70,5)$ & $376(43,2)$ \\
\hline Via pública & $0(0,0)$ & $49(14,2)$ & $63(14,8)$ & $21(23,9)$ & $133(15,3)$ \\
\hline
\end{tabular}




\begin{tabular}{lccccc} 
Outros & $3(21,4)$ & $215(62,5)$ & $100(23,5)$ & $5(5,7)$ & $323(37,1)$ \\
Total & 14 & 344 & 425 & 88 & 871 \\
& $34(79,1)$ & $28(82,4)$ & $62(80,5)$ & 0,72 & \\
\hline $\mathbf{2 0 1 4}$ & & & & & \\
\hline Escola & $0(0,0)$ & $11(4,5)$ & $13(4,0)$ & $3(5,3)$ & $27(4,1)$ \\
Residência & $19(73,1)$ & $151(61,9)$ & $231(70,9)$ & $36(63,2)$ & $437(66,9)$ \\
Via pública & $2(7,7)$ & $23(9,4)$ & $59(18,1)$ & $9(15,8)$ & $93(14,2)$ \\
Outros & $5(19,2)$ & $59(24,2)$ & $23(7,1)$ & $9(15,8)$ & $96(14,7)$ \\
Total & 26 & 244 & 326 & 57 & 653 \\
\hline
\end{tabular}

Quanto ao tipo de violência e a raça (Tabela 5), verificou-se que crianças e adolescentes da raça amarelo/indígena, tanto em 2013 quanto em 2014, foram vítimas, em sua maioria, de violência psicomoral e em segundo lugar, de estupro. Nas raças branca e preta a ordem se inverte, sendo o tipo mais comum o estupro e o segundo violência psicomoral. Na raça parda, em 2013 o tipo mais comum foi psicomoral, em segundo lugar o estupro, havendo inversão da ordem em 2014.

Tabela 5. Comparação entre a cor/raça e os tipos de violência sexual.

\begin{tabular}{|c|c|c|c|c|c|}
\hline \multirow{2}{*}{ Tipos de violência } & \multicolumn{4}{|c|}{ Cor / raça n (\%) } & \multirow[b]{2}{*}{ Total } \\
\hline & $\begin{array}{l}\text { Amarelo / } \\
\text { Indígena }\end{array}$ & Branco & Parda & Preta & \\
\hline \multicolumn{6}{|l|}{2013} \\
\hline Assédio sexual & $2(11,8)$ & $36(10,3)$ & $58(11,0)$ & $12(13,6)$ & $108(11,0)$ \\
\hline $\begin{array}{l}\text { Atentado violento } \\
\text { ao pudor }\end{array}$ & $0(0,0)$ & $27(7,7)$ & $25(4,8)$ & $4(4,5)$ & $56(5,7)$ \\
\hline Estupro & $5(29,4)$ & $141(40,3)$ & $187(35,6)$ & $37(42,0)$ & $370(37,8)$ \\
\hline Exploração sexual & $1(5,9)$ & $8(2,3)$ & $13(2,5)$ & $1(1,1)$ & $23(2,3)$ \\
\hline Pornografia & $0(0,0)$ & $6(1,7)$ & $12(2,3)$ & $2(2,3)$ & $20(2,0)$ \\
\hline Psicomoral & $9(52,9)$ & $132(37,7)$ & $230(43,8)$ & $32(36,4)$ & $403(41,1)$ \\
\hline Total & 17 & 350 & 525 & 88 & 980 \\
\hline \multicolumn{6}{|l|}{2014} \\
\hline Assédio sexual & $6(23,1)$ & $27(11,2)$ & $37(9,8)$ & $5(9,3)$ & $75(10,7)$ \\
\hline $\begin{array}{l}\text { Atentado violento } \\
\text { ao pudor }\end{array}$ & $3(11,5)$ & $9(3,7)$ & $28(7,4)$ & $1(1,9)$ & $41(5,8)$ \\
\hline Estupro & $7(26,9)$ & $111(45,9)$ & $169(44,6)$ & $24(44,4)$ & $311(44,4)$ \\
\hline Exploração sexual & $0(0,0)$ & $6(2,5)$ & $4(1,1)$ & $0(0,0)$ & $10(1,4)$ \\
\hline Pornografia & $0(0,0)$ & $4(1,7)$ & $8(2,1)$ & $3(5,6)$ & $15(2,1)$ \\
\hline Psicomoral & $10(38,5)$ & $85(35,1)$ & $133(35,1)$ & $21(38,9)$ & $249(35,5)$ \\
\hline Total & 26 & 242 & 379 & 54 & 701 \\
\hline
\end{tabular}

\section{Discussão}

De modo geral, os resultados do presente estudo evidenciam que a violência sexual praticada contra crianças e adolescentes no estado de Goiás nos anos de 2013 e 2014, predomina, em números absolutos, o estupro, seguido da violência psicomoral, sendo local mais comum no ambiente doméstico, a cor/raça sendo a parda e o sexo feminino como maioria das vítimas.

A violência sexual contra crianças e adolescentes é um tema que tem atraído a atenção de governantes, estudiosos, bem como da sociedade geral. Paralelamente a isso, apesar da reconhecida subnotificação, o número de casos notificados tem crescido cada vez mais, seja pela maior conscientização da população sobre a importância de denunciar a violência sexual ou dos profissionais de saúde em notificá-la, tanto casos suspeitos quanto confirmados. Verificam-se também inúmeras iniciativas e ações em saúde abordando a questão, demonstrando um aumento no reconhecimento da relevância dessa forma de violência.

A violência sexual nessa faixa etária tem consequências devastadoras e repercussões duradouras, pois está associada a transtornos psiquiátricos na vida adulta, como ansiedade, depressão, transtornos da conduta e aprendizagem, abuso de substâncias, hipersexualismo incompatível com a idade e transtornos alimentares. Também há relatos da sua associação com atraso no crescimento físico e no desenvolvimento da fala e linguagem, bem como dificuldades de manter laços afetivos e relacionamentos interpessoais ${ }^{11}$.

Este trabalho evidenciou elevada incidência de violência contra crianças e adolescentes no ambiente doméstico. Entretanto, pesquisa feita em 2011, nos Estados Unidos da América (EUA) ${ }^{12}$ relatou haver maior agressão em via pública. Estes dados sugerem que esse público se encontra em risco dentro e fora de casa, relacionando a violência na residência à violência da sociedade.

Há uma relação assimétrica de poder entre o agressor e a vítima. Segundo a literatura a maioria dos agressores é integrante do núcleo familiar, justificando assim a ocorrência na residência ser mais prevalente. A Violência é voltada ao mais vulnerável, não sendo exclusiva dos grupos sociais de menor poder de compra ou baixa escolaridade ${ }^{13}$. As relações familiares são complexas e esses atos de violência levam a rupturas da sustentação familiar, o que na maioria das vezes, não é de interesse do grupo familiar, motivando silêncio sobre os fatos ${ }^{14}$. Crianças e adolescentes, principalmente as primeiras, quando vítimas de violência sexual, não compreendem completamente, uma vez que não estão preparadas em termos de desenvolvimento, e, por conseguinte, tornam-se incapazes de informar seu consentimento. 
Quando a violência sexual ocorre por parte de pessoas conhecidas e/ou da confiança dessas crianças e adolescentes, há distorção da realidade e de sua visão de mundo. Uma vez que o ambiente que a priori seria seguro passa a se o palco privilegiado para o agressor. Qualquer tipo de violência, desde a sexual a física não pode ser enfrentada por estas crianças e adolescentes, contribuindo para uma deturpação da concepção de vida e mundo socialmente justos. $\mathrm{O}$ ato gera marcas permanentes, sejam elas biológicas ou psicológicas.

Em relação a raça/cor, o estudo demonstrou que houve um predomínio na raça parda, o que guarda íntima relação com a recuperação da identidade racial nos últimos anos no Brasil, principalmente nas últimas décadas. Os dados divulgados pelo setor de Pesquisa Nacional por Amostra de Domicílios (PNAD), realizada anualmente pelo Instituto Brasileiro de Geografia e Estatística (IBGE), há um significativo aumento da população que se declara negra ou parda. De acordo com a PNAD de 2006, os pardos compõem 79,782 milhões de pessoas, ou $42,6 \%$ da população do Brasil ${ }^{6}$.

Durante alguns anos o IBGE incluía pardos e negros na mesma categoria, o que foi recebido com críticas. A explicação dada pelo instituto é que a unificação dos tipos de raça estaria relacionada a condições econômicas parecidas, e não a valores culturais e sociais. Cabe ainda discussão para elucidar melhor a relação entre raça e violência sexual em crianças e adolescentes, além da constatação de haver uma maior quantidade de pessoas que se intitulam pardas no Brasil.

No quesito violência sexual, a literatura mundial aponta como um fenômeno feminino, sendo as meninas as principais vítimas de todos os tipos de violência sexual, independente da faixa etária. A vulnerabilidade é um fator essencial pra o entendimento da violência sexual contra crianças e adolescentes, já que as mesmas apresentam desvantagem física, emocional e social. Historicamente a incidência da violência sexual em mulher é maior e por esse motivo a discussão sobre gênero deve ser lembrada para compreender o fenômeno, de acordo coma campanha organizada pela organização Think Olga pela internet a média do primeiro assédio sofrido pelas mulheres foram em média de 9,7 anos.

A pesquisadora Heileth Saffioti no ano de 1997 considerou que a violência sexual não constitui uma pulsão irreprimível masculina, mas "uma questão de poder, afirmado a partir de relações assimétricas e dominadoras de homem contra mulher/meninas de adulto contra crianças e adolescentes. As discussões de aspecto social, cultural e de gênero abrangem discussões que muitas vezes são divergentes. O empoderamento da mulher na sociedade é um papel importante para o combate a esse tipo de violência.
O abuso psíquico é ainda pouco reconhecido e dificilmente é notificado, mas é tido na literatura como o mais comum. Como Diz Lídia Ester Lopes da Silva: A violência psicológica, eventualmente mais degradante que a violência física, é difícil de ser identificada em razão dos critérios para sua definição serem os limites da intimidade de convivência da vítima, essencialmente subjetivos.

De acordo com a médica e psicanalista de crianças e adolescentes, Luci Pfeiffer, do grupo interdisciplinar Defesa dos Direitos da Criança e do Adolescente - DEDICA do Hospital das Clínicas da Universidade Federal do Paraná, o abuso ou violência psíquica contra crianças e adolescentes se caracteriza pela prática, eventual ou rotineira, de atitudes ou ações que provoquem, conscientemente ou não, a humilhação, o sentimento de inferioridade ou de rejeição, que agridam moral ou eticamente, que tratem a criança ou o adolescente de forma preconceituosa, indiferente ou negligente e que, por fim, tem no abandono a sua forma mais cruel. A violência psicomoral é extremamente danosa quando praticada por indivíduos que compõe o grupo familiar, com quem a criança mantém vínculo afetivo e em quem confia.

A Declaração Universal dos Direitos Humanos, a Declaração Universal dos Direitos da Criança, a Constituição Brasileira e o Estatuto da Criança e do Adolescente trazem a questão da violência psicológica e preveem o direito da criança e do adolescente a ser protegido de tal forma de abuso. Infelizmente, o Código Penal Brasileiro, redigido em 1940, no entanto, não levam em consideração danos psíquicos ou emocionais, uma vez que trata os danos à saúde como "lesões corporais". Tornando-se, portanto, um obstáculo a medidas de proteção. Os laudos do Instituto Médico Legal (IML) têm como norma a lei de 1940, assim, em suas avaliações não levam em consideração danos psíquicos ou emocionais, necessários na avaliação para tipificação da agressão.

A principal limitação deste estudo encontrou-se na dificuldade decorrente do uso de dados secundários, provenientes das fichas de notificação dos casos de violência sexual. Havia muitas variáveis com consideráveis proporções de preenchimento dos campos 'Ignorado', 'Outros' e 'Em branco'. Destacamos que a presença de registros inadequados, muito comum em estudos desse tipo, não invalida os achados, porém recomenda cautela em sua interpretação.

Faz-se necessário que o profissional de saúde esteja apto para o correto preenchimento das Fichas de Notificação/Investigação Individual de Violência Doméstica, Sexual e/ou Outras Violências Interpessoais - FNIV - no momento do atendimento, alcançando, assim, maior acurácia dos dados. A melhoria da qualidade da informação, de como os dados foram colhidos 
e computados, é de fundamental importância para atribuir maior precisão aos processos de tomada de decisão. Ponderase ainda ser necessário qualificação e ampliação do Sistema de Vigilância de Violências e Acidentes - VIVA-tornando-o mais eficiente, atualizado e de fácil manipulação.

\section{Conclusão}

A violência sexual é um problema de saúde pública, considerado endêmico em vários locais. Para cada notificação de violência contra crianças e adolescentes, outras 10 a 20 agressões semelhantes não são registradas. $O$ presente estudo objetivou demonstrar as principais características relacionadas às vítimas, sendo estas com maior prevalência no sexo feminino, raça parda e a residência, o local de agressão mais comum. A violência sexual contribui significativamente para a redução da qualidade de vida não somente das vítimas, mas também de todos os atores envolvidos, podendo causar efeitos devastadores a curto, médio e longo prazo, principalmente no âmbito psicossocial.

Existe a necessidade de que os direitos previstos no ECA passem da literatura para a prática, com medidas mais efetivas. Observou-se a necessidade de acompanhar os casos junto ao Conselho Tutelar e à Vara Judicial, para saber qual desfecho dos casos estudados, bem como suas punições, para ter uma visão mais ampla e lançar medidas conjuntas que tenham maior eficiência.

Por meio deste estudo, detectou-se ainda a importância do Sistema de Vigilância de Violências e Acidentes -VIVA- na visibilidade desse agravo, evento de grande relevância para a saúde pública, assim como a importância da formação e sensibilização dos profissionais da saúde no preenchimento adequado do sistema de informação. É por conta do despreparo vivenciado por alguns profissionais de saúde na identificação da violência que ocorre a ausência da notificação, acarretando com isso a subestimação da morbidade por violência, pois muitos profissionais não dispõem de informações básicas que permitam diagnosticá-la a tempo.

\section{Referências Bibliográficas}

1. Delfino RK, Faria DS, Miranda MIF, Moraes RMB, Vasconcelos DMP. Violência sexual contra Crianças e Adolescentes - Perfil da vítima e do agressor em Porto Velho/RO. Rev. Bras. de Enfer. Ped. 2009;9(1):19-25.

2. Estatuto da criança e do adolescente [recurso eletrônico]: Lei n. 8.069, de 13 de julho de 1990, e legislação correlata. -13. ed. - Brasília: Câmara dos Deputados, Edições Câmara, 2015. 3. Brasil. 1a VIJ do Distrito Federal. Violência sexual contra crianças e adolescentes
4. Martins CBG. Abuso sexual na infância e adolescência: perfil das vítimas e agressores em município do Sul do Brasil. Texto Contexto Enfer. 2010;19(2):246-55.

5. Plano Nacional de Enfrentamento da Violência Sexual Infanto-Juvenil. 3. ed. Brasília: SEDH/DCA, 2002.

6. Waiselfisz JJ. Mapa da violência 2012: Crianças e Adolescentes do Brasil. Rio de Janeiro: Cebela, 2012.

7. Jesus APC. A violência sexual contra crianças e adolescentes no Brasil: da proteção jurídica às políticas públicas. 2015.

8. Brasil. Constituição de 1988. Constituição Federativa do Brasil. Brasília, DF: Senado Federal; 1988.

9. Pfeiffer EPS. Visão atual do abuso sexual na infância e adolescência. Artigo de revisão J. Pediatr. 2005;81(5):suppl.

10. Macdonald PL, Gardner RC. Type I error rate comparisons of post hoc procedures for I j Chi-Square tables. Educational and Psychological Measurement. 2000;60(5):735-54.

11. Facuri CO. Caracterização sociodemográficas e sintomas psíquicos de mulheres vítimas de violência sexual [dissertação]. Campinas: UNICAMP; 2012.

12. Breiding MJ, Smith SG, Basile KC, Walters ML, Chen J, Merrick MT. Prevalence and characteristics of sexual violence, stalking, and intimate partner violence victimization: national intimate partner and sexual violence survey, United States, 2011. MMWR Surveill Summ. 2014;63(8):1-7.

13. Fukumoto AECG, Corvino JM, Oobrich Neto J. Perfil dos agressores e das crianças e adolescentes vítimas de violência sexual. Rev. Ciênc. Ext. 2011;7(2):83.

14. Silva DG, Gava LL, Dell'Aglio DD. Sintomas e quadros psicopatológicos em supostas vítimas de abuso sexual: uma visão a partir da psicologia positiva. Atheia. 2013;(40):58-73. 